\title{
LE DOSSIER CONSTITUTIONNEL : Perspectives de Changement*
}

\section{Claude Ryan}

Ayant suivi avec attention l'évolution du dossier constitutionnel depuis le référendum tenu au Québec en octobre 1995, j'accueille avec plaisir l'occasion que nous fournit ce colloque de l'Institut de recherche en politiques publiques de mettre en commun les impressions et les conclusions que nous pouvons en retenir.

Rappelons à titre de points de repère les principaux développements suivants :

1) l'adoption par le Parlement fédéral, en décembre 1995, d'une résolution reconnaissant le caractère distinct $d u$ Québec et d'une loi obligeant le gouvernement fédéral à obtenir l'assentiment préalable du Québec avant de soumettre un projet de modification constitutionnelle à l'approbation du Parlement fédéral ${ }^{1}$;

2) le transfert aux provinces de la formation professionnelle de la maind'oeuvre;

3) l'abandon par le gouvernement fédéral de certaines conditions régissant l'utilisation des paiements de transfert en matière de santé, de services sociaux et d'enseignement postsecondaire;

4) la réduction des paiements de transfert fédéraux aux provinces dans le cadre du programme d'assain-

Texte publié dans la revue Options Politiques, jan-fév, 2000, 42. Republié ici avec l'autorisation d'Options Politiques de l'Institut de recherche en politiques publiques (IRPP), de maître Paul Ryan et de la famille Ryan.

1 P.L.C-110,Loi concernant les modifications constitutionnelles, $1^{\text {re }}$ sess., $35^{\mathrm{e}}$ Parl., 1996 (sanctionné le 2 février 1996), S.C. 1996, c. 1. issement des finances de gouvernement fédéral;

5) l'harmonisation des prestations fiscales pour enfants versées aux familles par les deux ordres de gouvernement;

6) l'abrogation de l'article 93 de la $L o i$ constitutionnelle de $1867^{2}$ dans son application au Québec;

7) la création du Fonds fédéral d'aide à l'innovation;

8) la création des bourses du millénaire;

9) la Déclaration de Calgary ${ }^{3}$;

10) l'Entente fédérale-provinciale sur l'union sociale canadienne;

11) l'Avis de la Cour suprême du Canada sur la sécession du Québec ${ }^{4}$.

Plusieurs de ces développements ont eu des effets nettement favorables sous l'angle des positions traditionnelles du Québec : ce fut notamment le cas du rapatriement de la formation professionnelle et de l'abrogation de l'article 93. D'autres ont donné lieu à des ententes, tels l'harmonisation des prestations fiscales pour enfants et le fonds d'aide à l'innovation. D'autres enfin, notamment la création des bourses du

(R.-U.), 30 \& 31 Vict., c. 3, art. 93, reproduit dans L.R.C. 1985, app. II, $\mathrm{n}^{\circ} 5$.

3 The Calgary Declaration (24 avril 1998), en ligne: Ontario Legislative Library $<$ http://www.ontla.on.ca/library/repository /mon/ 1000/10273337.html $>$.

4 Revoi relatifà la sécession du Québec, [1998] 2 R.C.S. 217, en ligne: IIJCan <http://www.canlii.org/ca/jug/csc/ 1998/1998csc63.html>. 
millénaire, l'Entente sur l'union sociale et les suites à apporter à l'Avis de la Cour suprême sur la sécession du Québec ont contribué à exacerber les rapports entre Québec et Ottawa. Quant à la Déclaration de Calgary, elle n'a eu, et pour cause, aucun impact significatif au Québec. De manière générale, la situation qui existait au lendemain du référendum de 1995 est demeurée la même. Le fossé constitutionnel qui sépare le Québec du reste du Canada depuis le rapatriement unilatéral de 1982 et l'échec de l'Accord du lac Meech est toujours là. Pour diverses raisons, aucun déblocage significatif ne semble devoir se produire dans les circonstances actuelles.

$\mathrm{Au}$ lieu de gémir sur cette situation contre laquelle, de manière immédiate, nous ne pouvons rien, j'y vois plutôt une invitation à réfléchir une fois de plus sur les désaccords qui sont à la racine des malaises entre le Québec et le reste du Canada. Je voudrais dans cette perspective soumettre à votre considération deux thèmes sur lesquels tout semble avoir été dit mais au sujet desquels des malentendus profonds subsistent toujours et semblent même en voie de s'accentuer. Le premier thème porte sur la dualité canadienne. Le second a trait au lien que nous, du Québec, établissons entre cette dualité et le rôle du Québec au sein de la fédération.

Dans son Avis sur la sécession du Québec, rendu public en août 1998, la Cour suprême énonçait quatre principes qui, à son avis, soustendent le système politique canadien, soit le principe fédéral, le principe démocratique, le constitutionnalisme et la règle de droit, et, enfin le respect des droits minoritaires ${ }^{5}$. Ainsi que le signalait peu après un juriste de l'Université Laval, Patrice Garant, la dualité linguistique ne figure pas parmi ces principes fondamentaux ${ }^{6}$. Comme la Cour suprême a pris soin de noter que son exposé n'avait pas la prétention d'être exhaustif, un commentaire me paraît s'imposer à ce sujet. Là où la Constitution affirme que le Canada compte deux langues officielles dotées chacune d'un statut d'égalité, le tribunal s'est plutôt borné à parler de droits minoritaires. Il est souvent question de diversité dans l'Avis de la

\footnotetext{
Ibid.

Patrice Garant, «Constitutionnalisme, primauté du droit et droit de sécession au sein d'une fédération», Communication présentée au Cinquième Congrès mondial de droit constitutionnel, Rotterdam, 12 au 16 juillet 1999 [non publiée].
}

Cour suprême, mais jamais explicitement de dualité. Ce langage confond deux ordres différents de réalité, soit d'une part le principe de l'égalité des deux langues, lequel consacre l'existence au Canada de deux communautés linguistiques principales et formant chacune une société d'accueil distincte, avec son corollaire obligé qui est le respect dû aux locuteurs de chacune des deux langues en ce qui touche l'accès à certains services publics dans les provinces où elles forment une minorité de langue officielle, et d'autre part le respect dû aux minorités en général et plus particulièrement aux catholiques et aux protestants en matière scolaire et aux communautés culturelles en général. Les mots diversité et dualité renvoient à deux ordres de réalité différents. En les confondant, on amoindrit dangereusement l'importance de la dualité.

En relisant le texte de la Cour suprême, il est difficile de ne pas se souvenir de la Déclaration de Calgary. Les auteurs de la Déclaration n'en finissaient pas d'exalter la diversité canadienne. Mais la dualité linguistique fut à juste titre considérée par les communautés francophones hors-Québec comme étant la parente pauvre du document. Tout ce qu'il restait du principe d'égalité dans la Déclaration de Calgary, hormis le paragraphe consacré au caractère " unique » du Québec, c'était une vague mention de la " vitalité des langues anglaise et française » dans un bref paragraphe où étaient également célébrés les mérites, incontestables par ailleurs, des peuples autochtones et des communautés culturelles.

A l'instar de Graham Fraser, qui consacrait récemment un article à ce sujet dans Le Devoir ${ }^{7}$,je m'inquiète d'une manière de voir maintenant fort répandue au Canada anglais, qui tend à minimiser la dualité linguistique et culturelle du Canada en ramenant cette dualité à une question de rapports entre une majorité anglophone et une minorité francophone. Cette attitude trouve sa contrepartie au Québec chez ceux pour qui il ne saurait exister entre francophones et anglophones que des rapports de force réglés en vertu de la même distinction réductrice. Selon l'esprit de la Constitution canadienne, c'est plutôt dans l'optique de l'égalité fondamentale des deux langues officielles du pays et non pas en termes de

Graham Fraser, «La dualité au programme» Le Devoir [de Montréal] (30 septembre 1999) A7. 
rapports entre majorité et minorité, qu'il faut poser le problème des rapports entre anglophones et francophones.

Il ne suffit pas cependant d'énoncer le principe de l'égalité des deux langues. Il faut aussi en proposer une interprétation généreuse et cohérente. Selon une interprétation légaliste, la Constitution, en vertu du principe d'égalité, garantit des droits particuliers aux locuteurs de l'une et l'autre langue dans certaines situations qu'a pris soin de définir le législateur, mais là s'arrête sa portée concrète. Selon une interprétation plus large, le législateur, tout en inscrivant dans la Constitution un certain nombre de droits précis, a aussi voulu y inscrire un principe vivant capable de donner lieu à des développements pouvant favoriser la réalisation concrète de l'égalité. Si l'on souscrit à cette deuxième interprétation, on doit être disposé non seulement à interpréter généreusement les textes juridiques mais aussi à rechercher et à promouvoir dans un esprit libéral les conditions les plus aptes à favoriser la réalisation concrète du principe d'égalité dans le plus grand nombre possible de situations. Dès qu'on le perçoit non pas comme un idéal abstrait mais dans son rapport nécessaire aux populations qui les parlent, le principe de l'égalité des deux langues a des implications incontestables pour le statut des minorités de langue officielle. D'ores et déjà, il connaît d'ailleurs de nombreuses applications dans la pratique des gouvernements et aussi dans la jurisprudence des tribunaux ainsi qu'on vient de le voir dans le cas de l'Hôpital Montfort ${ }^{8}$.

Mais le principe de l'égalité a également des implications incontournables quant au statut et au rôle du Québec dans la fédération canadienne. On trouve au Québec plus de $85 \%$ des francophones du Canada. Le Québec est en outre la seule province ayant une majorité francophone, les francophones y représentent en effet $83 \%$ de la population. Sans préjudice du rôle dévolu aux communautés francophones, aux gouvernements des autres provinces et au gouvernement fédéral, sans préjudice non plus de ses responsabilités envers sa communauté anglophone, le Québec est le foyer majeur et le point d'appui principal de la

8 Lalonde v. Ontario (Commission de restructuration des services de santé) (2001), 56 O.R.( $\left.3^{\mathrm{e}}\right) 505$ (CA),en ligne: IIJCan <www.canlii.org/on/cas/onca/2001 /2001onca10003.html\#sum>. vie française au Canada. En raison de ce trait qui le distingue nettement des autres provinces, le Québec - tout en ayant le statut juridique de province - n'est pas et ne peut pas être une simple province comme les autres au sein de l'ensemble canadien. Il est un intervenant majeur et très différent, sans lequel le principe d'égalité perd son sens. Il faut comprendre dans cette perspective l'insistance avec laquelle le Québec exige d'être associé à toute décision pouvant affecter les structures et les règles de conduite constitutionnelles. C'est aussi dans cette perspective que se situe la détermination des gouvernements du Québec, quelle que soit leur couleur politique, à s'acquitter eux-mêmes de leurs responsabilités dans les domaines plus directement reliés au caractère distinct de la société québécoise.

Suivant la même logique, le Québec insiste pour que son caractère distinct soit plus explicitement reconnu dans l'ordre constitutionnel canadien, et ce d'une manière qui ne soit pas uniquement symbolique mais qui donne ouverture à des effets pratiques. Cette revendication effarouche encore nombre d'observateurs. Elle est néanmoins le prolongement normal d'une longue histoire qui remonte à la Loi constitutionnelle de 1867 et qui a connu à l'époque contemporaine plusieurs développements intéressants (voir annexe 1). Ces développements, que je sache, n'ont créé d'injustice pour personne et n'ont aucunement mis en danger l'unité de pays. On n'en saurait dire autant de certaines rigidités, de certains revirements, de certaines décisions unilatérales dont les partenaires du Québec ont donné l'exemple à diverses reprises.

Quand je parle d'une reconnaissance qui aurait des effets pratiques, je ne pense pas à un chambardement radical dans le partage des compétences. Je m'opposai en 1991 au rapport Allaire parce que ce document proposait des changements majeurs dans le partage des pouvoirs sans que les auteurs en aient sérieusement mesuré les implications". Après avoir dirigé sept ministères différents pendant un séjour de neuf ans au sein du gouvernement du Québec et été témoin du double échec de Meech et Charlottetown, j'en étais venu à conclure en 1994 que le partage des

Jeau Allaire, Un Québec libre de ses choix, Montréal, Parti libéral du Québec, 1991. 
compétences établi par la Loi constitutionnelle de 1867 avait passablement bien résisté à l'épreuve du temps et qu'il ne m'apparaissait pas nécessaire de le modifier de fond en comble. Je demeure de cet avis. Je considère en conséquence qu'il serait plus sage dans les circonstances actuelles d'éviter de rouvrir tout le dossier du partage des compétences et de rechercher plutôt des améliorations ponctuelles dans la mesure où elles répondent à des besoins réels et démontrés. A titre d'exemples, j'avais retenu de mon séjour à la tête de sept ministères différents que des améliorations seraient souhaitables dans les secteurs du logement social, des affaires autochtones, des services correctionnels et du soutien à la recherche scientifique. A ces secteurs, d'autres ministres auraient sans doute ajouté la sécurité du revenu, la culture, le développement régional, les communications, l'aide à la petite et moyenne entreprise. Tous ces secteurs ont des liens directs avec le caractère distinct du Québec. La Commission Pepin-Robarts avait recommandé que de tels domaines soient objets de compétence partagée, avec prépondérance législative variable selon les champs et les modes d'intervention ${ }^{10}$. Cette avenue, dont les régimes de retraite canadien et québécois offrent un excellent exemple, fut trop vite écartée à l'époque. Il y aurait lieu de l'explorer de nouveau, en particulier en matière de sécurité du revenu et de culture.

Parmi les changements qui doivent être considérés comme impérieux et pressants, je situe en tout premier lieu l'encadrement du pouvoir fédéral de dépenser. Le pouvoir de dépenser m'apparaît comme une prérogative normale du gouvernement central dans une fédération. Je n'en conteste pas l'existence. Lorsque le gouvernement fédéral veut user de ce pouvoir pour instituer un programme à frais partagés dans un domaine relevant de la compétence provinciale, il ne devrait toutefois être habilité à le faire qu'après avoir obtenu le consentement d'une majorité satisfaisante de provinces et moyennant qu'une province, en particulier le Québec, n'ait pas à encourir de sanction financière si elle décide de ne

10 Canada, Royal Commissions and Commissions of Inquiry, Task Force on Canadian Unity: A Future Together, Hull, Canadian Government Publishing Centre, 1979; Task Force on Canadian Unity: A Time To Speak, Hull, Canadian Government Publishing Centre, 1979; et, Task Force on Canadian Unity: Coming To Terms, Hull, Canadian Government Publishing Centre, 1979. pas participer à un tel programme.

Le droit de veto du Québec m'apparaît toujours indispensable en raison de la dualité canadienne. La loi fédérale de 1995 a apporté à cet égard une amélioration appréciable pour le Québec mais seule une modification constitutionnelle pourra procurer en cette matière les garanties permanentes que le Québec exige à ce titre. Comme par ailleurs la loi de 1995 a introduit une rigidité excessive dans la procédure d'amendement, il faudra trouver une formule plus souple afin de pouvoir enchâsser éventuellement une nouvelle règle d'amendement dans la Constitution.

Nonobstant les changements survenus à notre époque dans la composition et la répartition de la population canadienne, la dualité linguistique et la réalité distincte du Québec demeurent les deux axes principaux autour desquels doit graviter toute recherche de solution au problème des rapports entre le Québec et le reste du Canada à l'intérieur du principe fédéral. On a tendance à disjoindre ces deux sujets, voire à les ériger l'un contre l'autre. Mais dans une perspective québécoise qui se veut fédéraliste, il n'est pas possible de les séparer. Toute solution durable devra prendre en compte l'un et l'autre volet. Le défi des prochaines années devra consister non pas à faire le silence sur ces réalités mais plutôt à chercher ensemble, pour les traduire dans nos arrangements constitutionnels et législatifs et dans nos pratiques administratives, des formules conciliables avec d'autres aspects de la réalité canadienne, notamment le renforcement de l'union économique, le statut des peuples autochtones, l'équilibre entre les régions et les attentes des communautés culturelles, qui doivent également retenir notre attention mais dont il n'était pas possible de discuter dans le cadre forcément limité de cet exposé.

\section{Claude Ryan}




\section{ANNEXE 1}

Exemples de reconnaissance du caractère distinct dans la tradition constitutionnelle et politique canadienne.

\section{A) DisPositions CONSTITUTIONNELLES}

1) la reconnaissance du droit civil québécois dans la $L o i$ constitutionnelle de 1867 (articles 93 et 94);

2) l'existence d'un régime linguistique spécial pour le Québec dans la $L o i$ constitutionnelle de 1867 (article 133);

3) l'obligation pour le gouvernement fédéral de choisir les juges des cours fédérales devant siéger au Québec parmi les membres du Barreau du Québec (Loi constitutionnelle de 1867, article 58);

4) l'insertion dans la Constitution en 1964 d'une disposition habilitant le Parlement fédéral à légiférer en matière de régimes de retraite mais spécifiant qu'en cas de conflit entre une loi fédérale et une loi provinciale, la loi provinciale aura prépondérance (article 94A);

5) l'insertion dans la Loi constitutionnelle de 1982 de dispositions garantissant le droit de retrait d'une province, moyennant juste compensation financière, à l'endroit de toute modification constitutionnelle entraînant un transfert de compétence des provinces en faveur du Parlement fédéral (articles 38, 3 et 40);

6) l'insertion dans la Charte canadienne des droits et libertés (1982) de dispositions affirmant l'égalité juridique du français et de l'anglais dans les travaux du Parlement, l'administration et les cours de justice fédérales, ete procurant l'accès à l'enseignement public primaire et secondaire dans leur langue aux enfants de communautés minoritaires de langue officielle (articles 16 à 23);

7) l'insertion dans la Loi constitutionnelle de 1982 d'une disposition respectant la souveraineté du Québec en ce qui touche les critères d'admission à l'enseignement en anglais (articles $59,2)$;

8) l'insertion dans la Charte canadienne des droits et libertés, au chapitre traitant de la liberté de circulation et d'établissement, de dispositions préservant une importante liberté de manoeuvre pour le Québec en matière de mesures visant à améliorer la situation d'individus défavorisés économiquement ou socialement (article 6);

9) l'insertion dans la $L o i$ constitutionnelle de 1982 d'une disposition garantissant que la composition de la Cour suprême, laquelle doit comprendre parmi ses neuf membres, trois juges en provenance du Québec, ne pourra être modifiée sans l'accord du Québec (article 41);

10) l'adoption en 1997 d'une modification constitutionnelle affranchissant le Québec des contraintes de l'article 93 de la $L o i$ constitutionnelle de 1867 et autorisant la création de commissions scolaires linguistiques.

\section{B) DisPOSITIONS LÉGISLATIVES ET/OU ADMINISTRATIVES}

1) l'adoption par le Parlement fédéral en 1995 d'une loi rendant obligatoire l'accord préalable du Québec avant la présentation au Parlement d'une proposition de modification de la Constitution; 
2) l'adoption par le Parlement fédéral en 1997 d'une résolution aux termes de laquelle le gouvernement fédéral et ses organismes sont tenus de prendre en compte la caractère distinct $d u$ Québec dans l'application des lois et la gestion des affaires du pays;

3) l'existence de réseaux publics de radio et de télévision en langue française desservant tout le pays mais ayant leur base principale au Québec et qui ont pu refléter et promouvoir avec une grande liberté la langue et l'essor de la culture au Québec;

4) l'existence depuis 1964 de la Loi fédérale sur les programmes établis en vertu de laquelle le Québec a pu prendre en charge sur son territoire, moyennant juste compensation financière, la gestion d'une trentaine de programmes pancanadiens à frais partagés;

5) les accords Gagnon-TremblayMcDougall sur l'immigration, en vertu desquels le Québec participe activement à la sélection des candidats à l'immigration et assume l'entière responsabilité de l'intégration des nouveaux arrivants en milieu québécois;

6) l'alternance de titulaires anglophones et francophones (les titulaires francophones émanant le plus souvent du Québec) dans les plus hautes fonctions du pays, notamment dans les postes de Gouverneur général, de Premier ministre fédéral, de Juge en chef de la Cour suprême, de Chef de l'étatmajor des Forces armées;

7) les arrangements concernant l'harmonisation des mesures fédérales et québécoises en matière de prestations fiscales pour enfants;

8) l'entente qui a permis le transfert au Québec de la formation professionnelle de la main-d'oeuvre;

9) l'entente qui a permis au Québec d'assumer la gestion entière du programme tripartite d'infrastructures;

10) les arrangements permettant l'implication directe du Québec, à titre de gouvernement participant, aux activités de la Francophonie internationale;

11) l'existence au Québec d'un système distinct de perception de l'impôt sur le revenu des particuliers. 